\title{
EMBARAZO Y ALCOHOL: CONOCIMIENTOS, ACTITUDES Y OPINIONES DE LOS PROFESIONALES SANITARIOS
}

\section{PREGNANCY AND ALCOHOL: KNOWLEDGE, ATTITUDES AND OPINIONS OF HEALTHCARE PROFESSIONALS}

\author{
Belén Charro Baena , Ainara Rivas Olivera² y Maria Raúl Xavier ${ }^{3}$ \\ ${ }^{1}$ Departamento de Psicología, Facultad de Ciencias Humanas y Sociales, Universidad Pontificia Comillas, España \\ ²Universidad Potificia Comillas, España
}

${ }^{3}$ Research Centre for Human Development (CEDH), Universidade Católica Portuguesa, Portugal

\section{Abstract}

Alcohol consumption during pregnancy represents an important risk for the fetus and is one of the main causes of the Fetal Alcohol Spectrum Disorders. Aims. To explore knowledge, attitudes and opin-ions of health professionals towards alcohol use during pregnancy. Method. Qualitative transversal study based on a phenomenological approach. Semi-structured interviews with 24 healthcare profes-sionals. Non-probability convenience sampling at public hospitals of the Community of Madrid. We performed a categorical and interpretive content analysis using QSR-NVIVO v.11 software. Results. Participants reported lack of knowledge about official guidelines and about Fetal Alcohol Spectrum Disorders, however they identified risk and consequences of drinking during pregnancy. Health professionals described the use of different approaches to advising pregnant women. Usually, partici-pants ask pregnant women only once about their alcohol use at the start of their pregnancy, and not follow-up with them. Participants have identified the need for better prevention and improve-ment/training on communication styles between professionals and clients. Conclusions. Results show the need to increase health professionals training about alcohol consumption during pregnancy and its consequences (including about Fetal Alcohol Spectrum Disorders) in order to improve quality of preventive actions

Keywords: alcohol, attitudes, healthcare professionals, opinions, pregnancy.

\section{Resumen}

El consumo de alcohol durante el embarazo representa un importante riesgo para el feto y es una de las principales causas de los Trastornos del Espectro Alcoholico Fetal. Objetivos. Explorar el conocimiento, actitudes y opiniones de los profesionales sanitarios sobre el uso de alcohol durante el embarazo. Método. Estudio cualitativo transversal basado en un enfoque fenoménológico. Realización de entrevistas semiestructuradas a 24 profesionales sanitarios. Muestreo no probabilístico por conveniencia en Hospitales de sanidad pública de la Comunidad de Madrid. Análisis categórico e interpretativo de contenido con ayuda del programa QSR-NVIVO v.11. Resultados. Se detecta cierto desconocimiento de las recomendaciones oficiales y de los Trastornos del Espectro Alcohólico Fetal, aunque se identifica el consumo de riesgo y las consecuencias para el feto. Diversidad de pautas a las gestantes sobre el consumo. No se suele incidir en la ingesta de alcohol en las entrevistas de seguimiento. Algunas gestantes minusvaloran el consumo. Propuesta de medidas para mejorar las acciones preventivas y la comunicación entre profesional y paciente. Conclusiones. Necesidad de incrementar la formación en los profesionales sanitarios sobre el consumo de alcohol, sus repercusiones durante el embarazo y sobre las Trastornos del Espectro Alcohólico Fetal para que pueda mejorar la prevención.

Palabras clave: actitudes, alcohol, embarazo, opiniones, profesionales sanitarios. 
El consumo de alcohol durante el embarazo representa un importante riesgo para el feto, ya que puede tener como resultado daños en su futuro funcionamiento cognitivo, social y emocional (France et al., 2010). Es una de las principales causas de déficits y problemas de neurodesarrollo en los niños, que en su conjunto se denominan Trastornos del Espectro Alcoholico Fetal (TEAF) y se asocian a daños físicos, malformaciones, disfunciones del comportamiento o discapacidades neurocognitivas (Coons, Watson, Yantzi, Lightfoot, \& Larocque, 2017).

Las guías clínicas recomiendan la abstinencia del alcohol durante el embarazo (Organización Mundial de la Salud (OMS), 2016), puesto que no se conoce un momento del embarazo en el que sea seguro consumir, ni un tipo de alcohol que no sea nocivo (Elek et al., 2013; Maier, \& West, 2001; Míguez, Magri, \& Suárez, 2009). Además, estas recomendaciones también promueven la abstinencia de alcohol cuando se está intentando concebir (OMS, 2016; Tough, Tofflemire, Clarke, \& Newburn-Cook, 2006). Pese a estas advertencias, muchas embarazadas siguen ingiriendo bebidas alcohólicas, incluso tras tomar conciencia de su estado. Algunos de los posibles motivos señalados tienen que ver con la poca y confusa información que tienen al respecto (López, 2013), o bien están relacionados con el estrés, la inmadurez, los casos de embarazo no planificado, el alcoholismo, la presión social y de grupo, la irresponsabilidad de la gestante y la depresión (Elek et al., 2013).

Se ha puesto en evidencia que los profesionales de la salud que trabajan en contacto directo con las mujeres embarazadas tienen un papel primordial en la prevención del TEAF (Scholin, Hughes, Bellis, Eriksson, \& Lorna, 2018). Sin embargo, se ha encontrado que en ocasiones proporcionan una información limitada sobre los riesgos del consumo de alcohol a la gestante, ya sea porque carecen de suficientes conocimientos sobre los efectos negativos del consumo durante el embarazo (France et al., 2010; Coons et al., 2017), desconocen las recomendaciones oficiales al respecto (Elliott, Payne, Haan, \& Bower, 2006), o bien porque consideran que preguntar sobre el consumo de alcohol puede provocar ansiedad, culpa e incluso ira en la mujer embarazada y temen estas respuestas (Payne et al., 2005). Por último, algunos resaltan la importancia de libertad de elección de la gestante respecto a su propio consumo (Coons et al., 2017).

El objetivo de este estudio es explorar las actitudes y opiniones de los profesionales sanitarios españoles sobre el uso de alcohol durante el embarazo. En concreto, se pretende examinar el nivel de información que tienen a este respecto, conocer cómo se informa a las gestantes sobre el consumo de alcohol y sus riesgos, saber qué medidas se toman y qué recursos emplean para ayudar a la embarazada, indagar en la actitud que perciben en las gestantes cuando se aborda esta problemática, las dificultades que encuentran los profesionales al plantearla y, por último, conocer las sugerencias que plantean para mejorar la información sobre las consecuencias del consumo de alcohol durante el embarazo.

\section{MÉTODO}

Diseño cualitativo transversal desde un enfoque fenomenológico, basado en el significado y experiencia de los participantes a través de una entrevista semiestructurada.

\section{Participantes}

Participaron 19 mujeres y 5 hombres de edades comprendidas entre los 23 y los 60 años $(M=32.04$; $D T=8.83)$, con un tiempo de experiencia ejerciendo su especialidad de entre 6 meses y 25 años $(M=6.29$ años; $D T=6.49)$. Los datos se presentan en la Tabla 1.

\section{Instrumentos}

Como instrumento se utilizó un guion semiestructurado sobre la temática en cuestión, que se construyó teniendo en cuenta la literatura revisada y la experiencia previa de las investigadoras, así como la discusión con dos equipos de investigación asociados a este proyecto en Francia y Portugal que están implementando un estudio similar. Las entrevistas se realizaron telefónicamente y se grabaron, una vez acordados día y hora, para ser posteriormente transcritas literalmente. El guion de entrevista se puede consultar en Anexo 1. 
Tabla 1. Características de los participantes

\begin{tabular}{|c|c|c|c|}
\hline Entrevista & Sexo & Especialidad & Tiempo de ejercicio profesional \\
\hline E1 & M & Enfermera en maternidad & 6 meses \\
\hline E2 & M & Ginecóloga & 7 años y 6 meses \\
\hline E3 & $\mathrm{H}$ & Residente de ginecología (R2) & 1 año y 6 meses \\
\hline E4 & $\mathrm{H}$ & Especialista en Enfermería Obstétrico-Ginecológica & 4 años \\
\hline E5 & M & Ginecóloga & 12 años \\
\hline E7 & M & Especialista en Enfermería Obstétrico-Ginecológica & 12 años \\
\hline E8 & M & Residente en ginecología y obstetricia (R4) & 3 años y 6 meses \\
\hline E9 & M & Residente en ginecología y obstetricia (R1) & 8 meses \\
\hline E10 & M & Ginecóloga y obstetra & 9 años \\
\hline E11 & M & Ginecóloga y obstetra & 8 años \\
\hline E13 & M & Enfermera en ginecología & 11 años \\
\hline E14 & M & Especialista en Enfermería Obstétrico-Ginecológica & 6 años \\
\hline E15 & M & Enfermera en maternidad & 6 años \\
\hline E16 & $\mathrm{H}$ & Ginecólogo & 9 años \\
\hline E17 & M & Especialista en Enfermería Obstétrico-Ginecológica & 21 años \\
\hline E18 & M & Especialista en Enfermería Obstétrico-Ginecológica & 25 años \\
\hline E19 & M & Residente en ginecología y obstetricia (R1) & 9 meses \\
\hline E20 & M & Residente en Enfermería Obstétrico-Ginecológica (R1) & 9 meses \\
\hline E21 & $\mathrm{H}$ & Residente en Enfermería Obstétrico-Ginecológica (R1) & 9 meses \\
\hline E22 & $\mathrm{H}$ & Residente en Enfermería Obstétrico-Ginecológica (R1) & 9 meses \\
\hline E23 & M & Residente en Enfermería Obstétrico-Ginecológica (R1) & 9 meses \\
\hline
\end{tabular}

Fuente: Elaboración propia

\section{Procedimiento}

Se presentó el proyecto a los Comités de Gerencia de 5 hospitales generales y públicos de la Comunidad de Madrid, solicitando la colaboración de los profesionales que cumplieran como criterios de inclusión la experiencia de trabajo en contacto directo con embarazadas o madres recientes (ginecólogos, obstetras, enfermeros, especialistas en enfermería obstétrico-ginecológica y residentes en dichas especialidades) y pertenecer al ámbito de la sanidad pública. Los comités de Gerencia de los hospitales aprobaron el estudio, pero comunicaron que no encontraban profesionales dispuestos a participar, por lo que se contactó directamente con algunos de ellos para, posteriormente, utilizar la metodología de bola de nieve (Ritchie y Lewis, 2003). El tamaño muestral atendió al criterio de saturación teórica (Creswell, 2007).

El estudio fue aprobado por el Comité Ético de la Universidad Pontificia Comillas. Se solicitó el consentimiento informado grabado en el momento de la entrevista y se ofreció un compromiso de confidencialidad a los participantes en el estudio. Previamente, se les informó sobre el objetivo del estudio, el tratamiento de la información, la voluntariedad de la participación y el anonimato de las entrevistas.

\section{Análisis de datos}

Se realizó un análisis categórico e interpretativo de contenido de las entrevistas, con ayuda del software de análisis cualitativo QSR-NVIVO v.11, que permite el cruce de los datos recogidos. A partir de la revisión bibliográfica, los objetivos de investigación y el guion de entrevista realizado, se confeccionaron unas categorías básicas con la que se codificaron todos los discursos obtenidos en torno a cinco dimensiones temáticas o nodos: (1) conocimiento de los profesionales de las consecuencias del consumo de alcohol durante el embarazo; (2) estándares de actuación e información a las gestantes; (3) conocimiento que tienen y actitud de las 
gestantes cuando se plantea el tema; (4) dificultades de los profesionales ante el abordaje del consumo de alcohol y (5) sugerencias de mejora. Estas categorías fueron descompuestas en subcategorías, constituyendo un sistema/árbol, por lo que los resultados se organizaron entorno a las cinco categorías principales o de primera generación, que a su vez se desdoblaron en categorías de segunda y tercera generación. Para conseguir la triangulación de investigadores (Ruiz Olabuénaga, 2008), reducir los sesgos y aportar consistencia y validez al análisis (Patton, 2002), una de las autoras procedió a la lectura de todas las entrevistas y realizó una primera identificación de los códigos y categorías de análisis de primera generación que se habían determinado previamente. Posteriormente, un segundo miembro del equipo revisó el sistema categorial, constituyendo las categorías de segunda y tercera generación. Cuando quedó consensuado entre las dos, se realizó la recodificación de todas las entrevistas. El sistema resultante fue revisado y discutido con el tercer miembro del equipo (Véase Anexo 2).

\section{RESULTADOS}

Se han encontrado diferencias en los discursos de los profesionales en función del sexo, del área de especialización y de los años de ejercicio profesional. En este sentido, las mujeres de todas las especialidades proporcionaban información más frecuentemente a la gestante y realizaban una tarea preventiva más acusada respecto al consumo de alcohol. Por otro lado, los especialistas en enfermería obstétrico-ginecológica (matronas) y enfermeras asistían de forma más cercana a las embarazadas y mostraban una mayor preocupación por los aspectos psicológicos del embarazo que aquellos especializados en ginecología/obstetricia. Por último, los participantes del estudio con menos experiencia laboral, principalmente residentes de enfermería en la especialidad obstétrico-ginecológica, mostraron mayor implicación e interés por el bienestar de las embarazadas.

El análisis del discurso a través de los nodos creados se describe en los siguientes apartados.

\section{Conocimiento de las consecuencias del consumo de alcohol durante el embarazo}

Aunque casi todos los profesionales sanitarios entrevistados (87.5\%) identificaron lo que constituye un consumo de riesgo, no hubo consenso respecto a la cantidad de alcohol que consideraban nocivo para la mujer o el feto. Mientras unos opinaban que cualquier uso se consideraría de riesgo, otros eran más permisivos. Algunos señalaron que el daño dependía de la dosis consumida, por lo que consideraban que no toda ingesta era perjudicial, o bien diferenciaban entre tipos de alcohol en sus recomendaciones. El discurso ha mostrado también un gran desconocimiento respecto a las bebidas "sin"; en este sentido, sólo algunos profesionales conocían la existencia de pequeños porcentajes de alcohol en estas bebidas y recomendaban la abstención durante la gestación. Los resultados han mostrado que varios participantes o bien desconocían las recomendaciones oficiales, o no las aplicaban.

También se ha encontrado una amplia variedad de respuestas al preguntar a los participantes por las posibles consecuencias del consumo de alcohol en el feto: Un $83.33 \%$ de los entrevistados mencionó malformaciones y problemas en el desarrollo y crecimiento, un $20.83 \%$ abortos, un $29.16 \%$ alteraciones del SNC y el neurodesarrollo y un $16.66 \%$ problemas comportamentales (irritabilidad, hiperactividad o agresividad). También se hizo mención a problemas visuales, auditivos y otras alteraciones inespecíficas, y un $58.33 \%$ de la muestra mencionó explícitamente el TEAF o el síndrome de abstinencia en el recién nacido. En cuanto a los efectos en la gestante, la mayoría señaló aspectos comunes con cualquier adulto consumidor de alcohol (62.5\%), como tolerancia, abstinencia, alcoholismo, problemas sociales, irritabilidad, problemas hepáticos, aumento del gasto cardíaco, factor de riesgo para el cáncer, problemas neurológicos, etc., mientras que sólo algunos aludieron a posibles accidentes provocados por el consumo (8.33\%) o problemas relacionados con el parto, incluyendo el síndrome de deprivación y peor control del dolor provocado por la tolerancia a la analgesia (4.16\%). Por último, un $12.50 \%$ mencionó las consecuencias psicológicas en caso de que la gestación no transcurriera adecuadamente.

Los ejemplos de los discursos en estas categorías se muestran en la Tabla 2. 
Tabla 2. Ejemplos de los discursos del nodo 1: Conocimiento de las consecuencias del consumo de alcohol durante el embarazo

\begin{tabular}{ll}
\hline Subnodo & Ejemplos de los discursos \\
\hline $\begin{array}{l}\text { Considera que todo consumo en } \\
\text { el embarazo es de riesgo }\end{array}$ & "Cualquier consumo, cualquier cantidad, por consenso, se considera de riesgo" (Entrevista 17). \\
Desconoce el consumo de riesgo & "Se sabe que realmente tiene que ser un consumo alto, un consumo diario a dosis altas, el típico consumo \\
& moderado de una copita de vino de manera casual no tiene por qué tener mayores consecuencias, pero un \\
& consumo constante, sobre todo el que sea constante, de manera rutinaria, pues a diario o todos los fines de \\
& semana durante todo el embarazo, sí que puede tener consecuencias; el problema es que no sabes con qué \\
& cantidad va a haber un síndrome alcohólico fetal" (Entrevista 6)
\end{tabular}

Diferencia entre tipos de alcohol más/menos nocivos

Diferencia entre cantidades y frecuencia de consumo para establecer el riesgo

Conoce las recomendaciones oficiales, pero no las aplica

Conoce las recomendaciones oficiales y las aplica

$\mathrm{Ni}$ conoce las recomendaciones oficiales ni las aplica

Conoce las consecuencias en el feto

Conoce las consecuencias en la madre

Conoce las consecuencias en ambos

Considera que hay suficiente información sobre el tema

Considera que no hay suficiente información sobre el tema

Soluciones para incrementar la información
"Un consumo seguro podría ser una copita de vino una vez al mes. Y nada de destilados, ni pensarlo" (Entrevista 22).

"Una UBE al día no es consumo de riesgo, beber más que eso sí" (E3).

“Es una pregunta un poco complicada, efectivamente. El alcohol es de estos tóxicos que no se pueden consumir, pero yo soy de las personas que opina que depende de la frecuencia [...], si es de manera esporádica, sí que se puede consumir. Y cuando digo alcohol, me refiero a una cerveza o una copa de vino muy circunstancialmente, y en ningún caso un cubata" (Entrevista 10).

"Al principio sí, y se le dice que en el embarazo no es segura ninguna cantidad de alcohol, ni siquiera de forma esporádica" (Entrevista 14).

“No, no pasa nada porque beba en ocasiones, ¿Quién soy yo para decirle a una mujer adulta qué puede o qué no puede beber?" (Entrevista 3).

“Hay muchísimas posibles consecuencias, para el feto las primeras que se me vienen a la cabeza son el síndrome alcohólico fetal, un bebé de bajo peso, problemas de visión y de audición, problemas en el comportamiento, muerte fetal antes del término del embarazo..." (Entrevista 4).

"Para la madre los mismos que para cualquier persona, con el agravante de que tiene un aumento, por ejemplo, del gasto cardíaco; entonces si tú tienes una taquicardia o alteraciones en la frecuencia cardíaca (que muchas veces se tienen con el alcohol), con un aumento del gasto pues es peor. O ya incluso los más banales, las alteraciones gástricas que se pueden tener con el alcohol pues en una embarazada se incrementan" (Entrevista 8).

"Para el feto las principales consecuencias son retrasos en el crecimiento, alteraciones en el neurodesarrollo y, por supuesto, el síndrome de neurodependencia. Y para la madre hay riesgo de parto prematuro y de mayor sangrado y complicaciones; también aumenta el riesgo de infecciones y disminuye su capacidad de control del dolor ya que beber alcohol aumenta su tolerancia a la analgesia y pueden llegar a sufrir síndrome de deprivación" (Entrevista 2).

"Yo creo que hay suficiente información. Información hay mucha. Que se le dé buen uso o no es otra cosa" (Entrevista 13).

“No hay suficiente información, tanto a nivel de la madre como de la población, e incluso de los profesionales me atrevería a decir" (Entrevista 17)

“Pues que se introdujera en los ambulatorios algún curso de información, y por supuesto, en la escuela, para los críos y los adolescentes no vendría mal. Indicarles que el alcohol es una droga y los peligros o el alcance que tiene. Y luego para las mujeres embarazadas pues en los talleres que hacemos a lo largo del embarazo meter una parte, igual que informamos sobre los hidratos de carbono, pues del alcohol; que ahora no se informa, sólo se hace una prohibición, "no tome alcohol y no fume". Yo les digo también las consecuencias físicas que puede tener para ella y para el feto" (Entrevista 18).

\section{Estándares de actuación e información acerca del consumo de alcohol a la gestante}

Los profesionales sanitarios entrevistados seguían los estándares de actuación fijados por los hospitales de la Comunidad de Madrid para las consultas durante la gestación. Un $66.67 \%$ de los participantes afirmó que en las primeras entrevistas se pregunta a las embarazadas si beben alcohol, incidiendo en cuánto y con qué frecuencia; un $4.17 \%$ dijo que, aunque esta pregunta se realizaba, no se incidía en la cantidad o la frecuencia del consumo. Un $8.33 \%$ expresó que plantearlo o no dependía del profesional, mientras que otro 8,33\% refirió no hacerlo nunca. Además, el $70.83 \%$ de los participantes expresó que esta pregunta forma parte de un protocolo estandarizado. El $4.17 \%$ dijo que, aunque está 
estandarizado, no siempre se realiza y el $12.5 \%$ dijo que no había nada estandarizado respecto a esto. En opinión de los encuestados, se da más importancia al consumo de tabaco. Se ha encontrado gran diversidad en las recomendaciones que los profesionales dan a las gestantes: desde consumo cero a consumo ocasional o ningún consejo al respecto del alcohol.

Respecto a qué medidas se toman en caso de que la gestante admitiese beber, aunque se tratase de un consumo moderado, se encontraron gran variedad de respuestas, aunque la mayoría aconsejaba dejar de beber - o al menos, reducir el consumo - y explicaba los efectos y consecuencias de la ingesta durante el embarazo. En cuanto a las ayudas o alternativas que se ofrecen a la gestante consumidora de alcohol, sólo el $4.16 \%$ de los entrevistados dijo hacerlo; de éstos, el 29.16\% refirió hacerlo sólo en los casos graves, el $20.83 \%$ señaló que no se les ofrecen alternativas, sólo información, y el 16.66\% mencionó la derivación a la unidad de Trabajo Social, al médico de familia o a grupos de apoyo; el 29.16\% restante no supo qué contestar a esta cuestión. Además, la mayoría de los profesionales entrevistados expresó que no existe ningún procedimiento estandarizado para estos casos de consumo en la embarazada y varios hicieron referencia a que no es una situación que se presente habitualmente.

En el caso de que la gestante negara beber, más de la mitad de los profesionales (54.16\%) indicó que no se reincide en ello durante el seguimiento del embarazo, aunque algunos señalaron que esta cuestión dependía del profesional o de si surgía algún problema durante la gestación (16.66\%). El 16.66\% afirmó que se vuelve a preguntar por el consumo, y un $12.5 \%$ de los entrevistados nunca se había encontrado en esta situación. El análisis del discurso mostró que generalmente los profesionales sanitarios no incidían en las distintas posibilidades de consumo que podían no haber quedado explicitadas como, por ejemplo, en el uso esporádico en momentos de celebración; tampoco exploraban las dificultades para no consumir que pudieran estar encontrando las bebedoras habituales. En la Tabla 3 se muestran ejemplos de los discursos.

\section{Conocimiento sobre el consumo de alcohol que tiene las gestantes y su reacción cuando se plantea el tema}

La mayoría de los profesionales entrevistados refirieron que, en general, las gestantes conocen bien las consecuencias del uso de alcohol durante el embarazo y cumplen la indicación de consumo cero, pero que en ocasiones tienen ideas equivocadas sobre el mismo, ya que algunas consideran que beber una cerveza o sólo de vez en cuando no es nocivo.

Tabla 3. Ejemplos de los discursos del nodo 2: Estándares de actuación e información a las gestantes

\begin{tabular}{|c|c|}
\hline Subnodo & Ejemplos de los discursos \\
\hline $\begin{array}{l}\text { Se sigue un } \\
\text { protocolo } \\
\text { estandarizado } \\
\text { para } \\
\text { preguntar }\end{array}$ & $\begin{array}{l}\text { "Cuando se le hace la anamnesis en la primera } \\
\text { visita obstétrica se le pregunta, por lo menos en } \\
\text { el hospital el formulario que tenemos pregunta } \\
\text { hábitos tóxicos, y en hábitos tóxicos entra } \\
\text { también el alcohol, debes preguntar por fumar y } \\
\text { beber. Sí, está estandarizado" (Entrevista 6). }\end{array}$ \\
\hline $\begin{array}{l}\text { Se pregunta, } \\
\text { incluyendo la } \\
\text { frecuencia y/o } \\
\text { cantidad }\end{array}$ & $\begin{array}{l}\text { "Sí, sí, se les pregunta por hábitos tóxicos, ya sea } \\
\text { el tabaco, el consumo de alcohol... la mayoría } \\
\text { suele responder que no, pero en caso afirmativo, } \\
\text { sí que se les pregunta por el hábito, por el tipo de } \\
\text { alcohol y la frecuencia con la que lo consumen, } \\
\text { así como si consumen otros tóxicos, algún tipo de } \\
\text { droga o alguna otra cosa, sí, a todas" (Entrevista } \\
\text { 12). }\end{array}$ \\
\hline $\begin{array}{l}\text { Sólo se } \\
\text { pregunta en la } \\
\text { primera } \\
\text { consulta }\end{array}$ & $\begin{array}{l}\text { "Sí que se les pregunta, pero sólo en la primera } \\
\text { consulta, suelen decir que no y no se hace un } \\
\text { seguimiento" (Entrevista 2). }\end{array}$ \\
\hline $\begin{array}{l}\text { No hay un } \\
\text { protocolo } \\
\text { estandarizado } \\
\text { o lo } \\
\text { desconoce }\end{array}$ & $\begin{array}{l}\text { "No está estandarizado, se debe hacer, pero yo } \\
\text { creo que no todo el mundo lo pregunta" } \\
\text { (Entrevista 11). }\end{array}$ \\
\hline $\begin{array}{l}\text { Se pregunta } \\
\text { por el } \\
\text { consumo de } \\
\text { tóxicos en } \\
\text { general }\end{array}$ & $\begin{array}{l}\text { "Sólo se pregunta por hábitos. Si bebe, fuma... y y } \\
\text { ya está; es si o no. De hecho, en alcohol creo que } \\
\text { no te da la opción ni a poner cantidades, cosa } \\
\text { que si se puede en tabaco" (Entrevista 19). }\end{array}$ \\
\hline $\begin{array}{l}\text { Se centran en } \\
\text { el consumo } \\
\text { de tabaco }\end{array}$ & $\begin{array}{l}\text { "Hay que preguntar por los hábitos tóxicos de } \\
\text { manera general, pero se suele preguntar por el } \\
\text { tabaco, le damos más importancia al tabaco, } \\
\text { dejamos el alcohol más de lado" (Entrevista 9). }\end{array}$ \\
\hline $\begin{array}{l}\text { Son más } \\
\text { permisivos } \\
\text { que con otras } \\
\text { sustancias } \\
\text { tóxicas }\end{array}$ & $\begin{array}{l}\text { "El alcohol está muy aceptado en nuestra } \\
\text { tradición: La cerveza, la copa de vino... En nuestra } \\
\text { sociedad el consumo de alcohol forma parte de } \\
\text { nuestra cultura, y esto permite que seamos más } \\
\text { permisivos que con respecto a otras sustancias" } \\
\text { (Entrevista 7). }\end{array}$ \\
\hline No pregunta & $\begin{array}{l}\text { "Nunca he tratado este tema con ellas [...] O bien } \\
\text { porque doy por hecho que no hay un consumo } \\
\text { de alcohol, que parece ser que quiero }\end{array}$ \\
\hline $\begin{array}{l}\text { Si la gestante } \\
\text { admite beber, } \\
\text { explora los } \\
\text { motivos de } \\
\text { consumo }\end{array}$ & $\begin{array}{l}\text { imaginarme que va todo bien, o que... no sé, pero } \\
\text { no lo abordo" (Entrevista 21). } \\
\text { "Yo pregunto por qué lo toma, según la } \\
\text { explicación que me dé intento decirle maneras de } \\
\text { ir reduciendo al mínimo ese consumo" (Entrevista } \\
\text { 18). }\end{array}$ \\
\hline
\end{tabular}

Fuente: Elaboración propia. 
Tabla 3. Ejemplos de los discursos del nodo 2: Estándares de actuación e información a las gestantes (continuación)

\begin{tabular}{|c|c|}
\hline Subnodo & Ejemplos de los discursos \\
\hline $\begin{array}{l}\text { Si la gestante } \\
\text { admite beber, } \\
\text { ofrece ayudas } \\
\text { o alternativas }\end{array}$ & $\begin{array}{l}\text { “Depende del grado de consumo, de la mujer y } \\
\text { cómo reciba el mensaje; puede ser simplemente } \\
\text { consejo [...] o, si ya hay un problema más } \\
\text { importante, como alcoholismo, coordinación con } \\
\text { especializada; a veces también con el trabajador } \\
\text { social y con el médico de familia" (Entrevista 17). }\end{array}$ \\
\hline $\begin{array}{l}\text { Si la gestante } \\
\text { admite beber, } \\
\text { aconseja que } \\
\text { no beba }\end{array}$ & $\begin{array}{l}\text { "Lo primero es informarla sobre los riesgos que } \\
\text { conlleva el consumo de alcohol para el feto y } \\
\text { decirle que debería dejarlo. Y, en función de la } \\
\text { disposición de la mujer, se le proponen diferentes } \\
\text { alternativas. Si es una mujer que no está nada } \\
\text { dispuesta a dejar de beber alcohol, lo que le } \\
\text { puedes recomendar es que reduzca su consumo } \\
\text { al mínimo posible. Si te dice que no sabía los } \\
\text { riesgos que tenía o que sí que está dispuesta a } \\
\text { dejarlo, pues simplemente le recomiendas que no } \\
\text { beba alcohol y se apunta que consume alcohol } \\
\text { para volver a preguntar en la siguiente cita" } \\
\text { (Entrevista 23). }\end{array}$ \\
\hline
\end{tabular}

Fuente: Elaboración propia

Las mujeres suelen reaccionar con naturalidad al abordar el tema del alcohol, aunque algunos profesionales añadieron que en ocasiones se sorprenden de la pregunta porque les resulta obvio el cese del consumo durante la gestación. Muchos entrevistados afirmaron que es habitual que algunas mujeres mientan respecto al consumo de alcohol, tratando de minimizarlo, especialmente las que beben habitualmente. Son aquellas que cuando se plantea el tema reaccionan defensivamente, con vergüenza o culpa, intentando justificarse. Se muestran ejemplos de este nodo en la Tabla 4.

\section{Dificultades de los profesionales}

La mayor parte de los profesionales refirió no tener ninguna dificultad a la hora de tratar el tema del consumo de alcohol con las gestantes. Sólo algunos expresaron que en ocasiones les cuesta abordar el tema por temor a emitir un juicio de valor, a generar ansiedad a la embarazada o, en casos de alcoholismo, debido a todas las complicaciones y aspectos asociados que presenta. Entre estos, el problema más señalado (95.83\%) estaba relacionado con la falta de tiempo en las consultas para poder abordar toda la problemática que pueda repercutir en la gestación y en el feto. El $45.83 \%$ de los entrevistados consideró que sus conocimientos sobre el consumo de alcohol durante el embarazo eran limitados y que se trataba de un tema tabú y el $41.66 \%$ afirmó que preguntar sobre el consumo de alcohol puede provocar que las embarazadas se sientan juzgadas. Los ejemplos del discurso de este nodo aparecen en la Tabla 5.

Tabla 4. Ejemplos de los discursos del nodo 3: Reacciones de las gestantes y conocimientos sobre el tema

\begin{tabular}{|c|c|}
\hline Subnodo & Ejemplos de los discursos \\
\hline $\begin{array}{l}\text { Conocen los } \\
\text { efectos y } \\
\text { consecuencias } \\
\text { del consumo } \\
\text { de alcohol }\end{array}$ & $\begin{array}{l}\text { "Cuando la gestante llega a la consulta sí que está } \\
\text { dispuesta a hacer todo lo que pueda por proteger } \\
\text { a su bebé. Entonces suelen estar bastante } \\
\text { abiertas a: Si no puedo tomar absolutamente } \\
\text { nada, no lo tomo" (Entrevista 20). }\end{array}$ \\
\hline $\begin{array}{l}\text { No conocen } \\
\text { los efectos y } \\
\text { consecuencias }\end{array}$ & $\begin{array}{l}\text { "Si profundizas un poco más y les dices que los } \\
\text { fines de semana tampoco deben beber ni una } \\
\text { cerveza, sí que te dicen: ah, ¿ni siquiera eso?" } \\
\text { (Entrevista 17). }\end{array}$ \\
\hline $\begin{array}{l}\text { Reaccionan } \\
\text { con } \\
\text { naturalidad }\end{array}$ & $\begin{array}{l}\text { "Responden bien, con naturalidad, además saben } \\
\text { que todo es confidencial y de ahí no va a salir." } \\
\text { (Entrevista 15). }\end{array}$ \\
\hline $\begin{array}{l}\text { Reaccionan "a } \\
\text { la defensiva" }\end{array}$ & $\begin{array}{l}\text { "Tiene pinta de que saben que no lo tienen que } \\
\text { hacer y, cuando tratamos el tema, se muestran a } \\
\text { la defensiva" (Entrevista 10). }\end{array}$ \\
\hline Mienten & $\begin{array}{l}\text { "Creo que todas las mujeres que beben mienten, } \\
\text { no me creo que en } 8 \text { años sólo me haya } \\
\text { encontrado una mujer que me ha contestado que } \\
\text { bebía más de la cuenta. Una de dos: o yo no sé } \\
\text { formular la pregunta de forma correcta, o algunas } \\
\text { mienten" (Entrevista 11). }\end{array}$ \\
\hline
\end{tabular}

Fuente: Elaboración propia.

Tabla 5. Ejemplos de los discursos del nodo 4: Dificultades de los profesionales ante el abordaje del consumo de alcohol

\begin{tabular}{|c|c|}
\hline Subnodo & Ejemplos de los discursos \\
\hline $\begin{array}{l}\text { Dificultades } \\
\text { porque temen } \\
\text { emitir juicios } \\
\text { de valor }\end{array}$ & $\begin{array}{l}\text { Yo creo que, al final, todos tenemos un poco de } \\
\text { miedo de emitir un juicio de valor y que luego no } \\
\text { sea así" (Entrevista 14). }\end{array}$ \\
\hline $\begin{array}{l}\text { Dificultades } \\
\text { porque temen } \\
\text { encontrase } \\
\text { con casos de } \\
\text { alcoholismo }\end{array}$ & $\begin{array}{l}\text { "Si se trata de una persona con un problema } \\
\text { alcohólico puede llegar a ser muy complicado, } \\
\text { porque además tienes que tratar el problema que } \\
\text { tenga la persona" (Entrevista 1). }\end{array}$ \\
\hline $\begin{array}{l}\text { Dificultades } \\
\text { por la falta de } \\
\text { tiempo en } \\
\text { consulta }\end{array}$ & $\begin{array}{l}\text { "Normalmente la entrevista médica es muy } \\
\text { limitada, el tiempo que tenemos, entonces al } \\
\text { preguntar por los hábitos tóxicos si la paciente } \\
\text { niega en ese momento el consumo de alcohol, no } \\
\text { vamos más allá" (Entrevista 12). }\end{array}$ \\
\hline $\begin{array}{l}\text { Dificultades } \\
\text { porque } \\
\text { consideran } \\
\text { que es un } \\
\text { tema tabú }\end{array}$ & $\begin{array}{l}\text { "Las embarazadas con un problema de } \\
\text { alcoholismo... me resulta duro porque son } \\
\text { problemas generalmente crónicos, generalmente } \\
\text { tabú, generalmente se acompañan de problemas } \\
\text { sociales y familiares, a veces con otros problemas } \\
\text { de adicción. Son problemas muy complejos y, en } \\
\text { ese sentido, me resulta duro" (Entrevista 17). }\end{array}$ \\
\hline $\begin{array}{l}\text { No presentan } \\
\text { dificultades }\end{array}$ & $\begin{array}{l}\text { "Yo personalmente no tengo ninguna dificultad } \\
\text { para tratar este tema con las embarazadas, } \\
\text { aunque es cierto que no puedo controlar que sean } \\
\text { honestas" (Entrevista 4). }\end{array}$ \\
\hline
\end{tabular}

Fuente: Elaboración propia. 


\section{Sugerencias para mejorar la información sobre embarazo y consumo de alcohol}

Para paliar la falta de información actual sobre el consumo de alcohol en el embarazo y sus consecuencias, el $87.5 \%$ de los profesionales reflejó la necesidad de realizar educación para la salud en la población y campañas en medios de comunicación, fomentar la inclusión de esta temática en cursos de embarazo y grupos de apoyo, además de proporcionar esa información desde atención primaria y realizar consultas preconcepcionales. Asimismo, propusieron medidas para mejorar la comunicación entre profesional y paciente respecto a este tema. Destacaron la importancia de no juzgar y de generar una relación de confianza con la embarazada, así como hicieron referencia a la necesidad de que los profesionales estén más formados, tanto en el tema del consumo de alcohol en el embarazo como en habilidades de comunicación (58.33\%). Por último, algunos propusieron establecer un protocolo, dar folletos en caso de dificultad con la comunicación oral, o incluso derivar a otro profesional (12.5\%). Los ejemplos del discurso de este nodo aparecen en la Tabla 6.

Tabla 6. Ejemplos de los discursos del nodo 5: Sugerencias para mejorar la información sobre el consumo de alcohol y el embarazo

\begin{tabular}{|c|c|}
\hline Subnodo & Ejemplos de los discursos \\
\hline $\begin{array}{l}\text { Campañas en los } \\
\text { medios de } \\
\text { comunicación }\end{array}$ & $\begin{array}{l}\text { "Campañas y demás, lo típico, hay temas que } \\
\text { quizá son más relevantes, pero es más difícil } \\
\text { llevar a cabo una campaña Todo lo que sea } \\
\text { llevar a cabo publicidad para darlo a conocer" } \\
\text { (Entrevista 24). }\end{array}$ \\
\hline $\begin{array}{l}\text { Información } \\
\text { específica para } \\
\text { gestantes: } \\
\text { trípticos, avisos en } \\
\text { bebidas, ámbito } \\
\text { sanitario }\end{array}$ & $\begin{array}{l}\text { “Hojas informativas y trípticos. Que su } \\
\text { ginecólogo o su matrona hablen de todo este } \\
\text { tema y den toda esa información la primera } \\
\text { vez que acuden al centro de salud, hacerlo al } \\
\text { inicio del embarazo” (Entrevista 1). }\end{array}$ \\
\hline $\begin{array}{l}\text { Mejoras en la } \\
\text { comunicación } \\
\text { profesional } \\
\text { sanitario/gestante }\end{array}$ & $\begin{array}{l}\text { "Yo creo que nosotros, como profesionales, } \\
\text { deberíamos tener más formación, no sólo en } \\
\text { las consecuencias, para poder transmitirlas } \\
\text { mejor, sino también en comunicación, porque } \\
\text { a veces se nos da un poquito peor" (Entrevista } \\
\text { 14). }\end{array}$ \\
\hline $\begin{array}{l}\text { Incrementar la } \\
\text { formación sobre } \\
\text { alcohol en los } \\
\text { profesionales } \\
\text { sanitarios }\end{array}$ & $\begin{array}{l}\text { “Creo que los profesionales deberíamos estar } \\
\text { más formados en cuanto a este tema, y luego } \\
\text { yo creo que debería haber mucha más } \\
\text { información y educación con respecto a estas } \\
\text { prácticas en la población" (Entrevista 9). }\end{array}$ \\
\hline
\end{tabular}

Fuente: Elaboración propia.

\section{DISCUSIÓN}

El análisis del discurso de los participantes ha mostrado diferencias en el grado de preocupación por el bienestar psicológico de las gestantes y las actuaciones preventivas frente al consumo de alcohol. De esta manera, los más implicados eran los especialistas en enfermería obstétrico-ginecológica o general, probablemente porque el seguimiento que realizan de las embarazadas es más continuo y se orienta más a dar pautas y a aportar información relacionada con la gestación, mientras que el seguimiento de los especialistas en ginecología se centra en comprobar el adecuado desarrollo del embarazo. También se ha hallado mayor interés en los aspectos psicológicos en aquellos participantes con menor tiempo de experiencia laboral. Esto podría deberse al entusiasmo asociado al inicio profesional, por un lado, o a que la formación actual ponga más énfasis en estos aspectos que la formación que se recibía en el pasado, pero no se han encontrado datos al respecto en la literatura revisada para poderlo contrastar. Por último, se ha encontrado que las participantes mujeres asistían de manera más cercana a las gestantes, les proporcionaban más información y realizaban con más frecuencia tareas preventivas frente al consumo de alcohol. Este resultado es congruente con los datos obtenidos por otros trabajos que muestran que las mujeres se centran más que los hombres en cuidar la relación médico-paciente (Haidet et al, 2002), se orientan más hacia los aspectos psicosociales y hacia las actividades preventivas (Delgado, López-Fernández, \& Luna, 2001).

Los resultados de este estudio confirman los datos obtenidos por trabajos previos al encontrar que muchos de los profesionales entrevistados no dan indicaciones siguiendo las recomendaciones de las guías clínicas actuales (por ejemplo, Elliot et al, 2006; Payne et al, 2005). Esto provoca disparidad en los consejos a las embarazadas, lo que puede causar confusión y conducir a la gestante a sacar sus propias conclusiones, como ya se ha señalado anteriormente (Elek et al, 2013). También se ha encontrado que desconocen la existencia de un protocolo de actuación para casos de alcoholismo o ingesta excesiva durante el embarazo. Además, se han hallado actitudes muy permisivas respecto al consumo de alcohol de las gestantes en varios profesionales, como ya ocurrió en un trabajo realizado en la Comunidad Valenciana (Alcantud, \& Alonso, 2012). 
Se ha encontrado que la mayoría de los participantes conoce las consecuencias del consumo de alcohol en el feto, aunque no todos hicieron referencia al TEAF, a pesar de su importancia refrendada por la literatura (Coles et al, 2016; Coons et al, 2017). Se ha detectado que algunos entrevistados no estaban concienciados sobre su responsabilidad a la hora de aconsejar a las embarazadas para evitar esta y otras problemáticas derivadas del consumo de alcohol, por lo que se hace evidente la necesidad de trabajar en la mejora de la actitud de los profesionales sanitarios hacia las actividades preventivas (OMS, 2016).

Aunque los participantes dijeron seguir los protocolos de los hospitales de la Comunidad de Madrid para explorar el uso de tóxicos durante la gestación, varios señalaron que se da más importancia al tabaco y que en muchas ocasiones no se indaga sobre el consumo de alcohol. Se ha detectado que, generalmente, cuando tratan el tema no inciden en los riesgos y consecuencias, sino que aconsejan no consumir. En este sentido, estudios anteriores han mostrado que informar a las gestantes sobre las consecuencias negativas del alcohol para el feto resulta más efectivo para frenar la ingesta que recomendar la abstinencia únicamente (Elek et al, 2013). Por otro lado, en los casos de embarazadas con consumo problemático de alcohol, la mayoría sólo ofrecía alternativas en los casos graves. En esta línea, algunos trabajos han señalado que las matronas consideraban que este aspecto excedía sus funciones (Benito López et al, 2016).

Los profesionales refirieron que las mujeres suelen conocer la recomendación de abstinencia del alcohol durante el embarazo, pero señalaron que suelen ocultar o minimizar su consumo, sobre todo cuando es problemático. En estudios anteriores se ha señalado que las mujeres suelen racionalizar su consumo durante el embarazo y justificarlo por el estrés (Fletcher, May, Seedat, Sikkema, \& Watt, 2018), consideran que algunos tipos de alcohol son menos dañinos (Dumas et al, 2018) o están confusas respecto al consumo de riesgo (Howlett et al, 2017). Otros trabajos ya habían constatado que las gestantes no expresan los datos reales en cuanto a cantidad y frecuencia (Da Rosa et al, 2016), y proponen utilizar biomarcadores de exposición fetal al alcohol como dato más objetivo (Manich et al, 2012).
La principal dificultad que reflejaron los participantes fue la limitación de tiempo de consulta, que les impide abordar todos los temas que les parecen importantes y les fuerza a priorizar. Esto concuerda con los datos obtenidos en otros estudios (Coles et al, 2016; Ramos-Morcillo, Ruzafa-Martínez, Fernández-Salazar, del-Pino-Casado, \& Armero, 2014), en los cuales se encontró la falta de tiempo como uno de los principales problemas que impiden realizar labores de prevención. También señalaron que en ocasiones les resultaba problemático abordar el tema del consumo del alcohol con las gestantes sin caer en juicios de valor. Ya se había puesto de relieve con anterioridad que las cuestiones sobre el consumo de alcohol que realizan las matronas, a pesar de su eficacia, crean incomodidad tanto en ellas como en las gestantes (Watkins et al, 2015).

Respecto a las sugerencias para informar mejor sobre las consecuencias del consumo de alcohol a las embarazadas, se propuso abordar el tema desde atención primaria y en consultas preconcepcionales. También, como habían sugerido otros trabajos (Elek et al, 2013), se planteó la necesidad de realizar campañas de prevención y promoción de la salud en medios de comunicación, entre otros. Además, también se mencionó la necesidad de mejorar la formación de los profesionales sanitarios, tanto en lo referido a las consecuencias del consumo de alcohol para la madre y el feto, como en habilidades específicas de comunicación con los pacientes, al igual que ya había sido puesto en evidencia en estudios anteriores (Nebot et al, 2007).

\section{Conclusiones}

Los resultados han puesto en evidencia cierto desconocimiento entre los profesionales sanitarios y las mujeres gestantes de las recomendaciones oficiales y de las consecuencias del consumo del alcohol en el feto y en la embarazada. Estos hallazgos sugieren la necesidad de incrementar la formación en el sector sanitario y en la población general para mejorar las actuaciones preventivas.

Este estudio presenta algunas limitaciones. En primer lugar, la localización geográfica de los participantes y la prevalencia del sexo femenino hace que los discursos recogidos no sean generalizables. Por otro lado, en futuros trabajos se deberá depurar el guion de entrevista, ya que en alguna ocasión la manera de preguntar podía inducir la respuesta de los participantes; 
también se debería alterar el orden y realizar primero las preguntas menos específicas, para pasar luego las más específicas. No obstante, hay que señalar como aspecto positivo que se realizó la triangulación de investigadores y se llegó a la saturación teórica, lo que ha permitido ilustrar las opiniones y actitudes de los facultativos frente al consumo de alcohol durante la gestación partiendo de sus propios discursos.

\section{Reconocimientos}

A todos los profesionales sanitarios que han colaborado en este trabajo.

\section{Conflicto de intereses}

Las autoras declaran que no existen conflictos de intereses y que el estudio no ha recibido ningún tipo de financiación.

\section{REFERENCIAS}

Alcantud, F., \& Alonso, Y. (2012). Actitudes y conocimientos sobre las consecuencias del consumo de bebidas alcohólicas durante la gestación y la lactancia en profesionales socio-sanitarios de la ciudad de Valencia. Revista Española de Drogodependencias, 37(4), 411-29.

Benito López, V. E., Bolaños Gallardo, E., Redondo Martín, S., \& Maderuelo Fernández, J. A. (2016). Opiniones de los y las profesionales de Atención Primaria sobre el consumo de alcohol en mujeres: Barreras percibidas para la intervención. Revista Española de Drogodependencias, 41(3), 72-83.

Coles, C. D., Gailey, A. R., Mulle, J. G., Kable, J. A., Lynch, M. E, \& Jones, K. L. (2016). A comparison among 5 methods for the clinical diagnosis of fetal alcohol spectrum disorders. Alcoholism: Clinical and Experimental Research, 40, 1000-1009. doi: 10.1111/acer.13032

Coons, K., Watson, S., Yantzi, N., Lightfoot, N., \& Larocque, S. (2017). “No Alcohol Is Recommended, But...": Health Care Students' Attitudes About Alcohol Consumption During Pregnancy. Global Qualitative Nursing Research, 3, 1-12. doi: 10.1177/2333393617707663

Creswell, J. (2007). Qualitative Inquiry and Research Design - choosing among five approaches. London: Sage

Delgado, A., López-Fernández, L., \& Luna, J. (2001). Ser médico o médica marca diferencias en la práctica asistencial. Atención Primaria, 28(4), 219-226. doi: 10.1016/S0212-6567(01)78938-X

Dumas, A., Toutain, S., Hill, C, \& Simmat-Durand, L. (2018). Warning about drinking during pregnancy: lessons from the French experience. Reproductive Health, 15(1), 20. doi:10.1186/s12978-018-0467-x

Elek, E., Harris, S., Squire, C., Margolis, M., Weber, M., Dang, E., \& Mitchell, B. (2013). Women's knowledge, views, and experiences regarding alcohol use and pregnancy: opportunities to improve health messages. American Journal of Health Education, 44(4), 177-190. doi: 10.1080/19325037.2013.768906.

Elliott, E., Payne, J., Haan, E., \& Bower, C. (2006). Diagnosis of fetal alcohol syndrome and alcohol use in pregnancy: A survey of paediatricians' knowledge, attitudes and practice. Journal of Paediatrics And Child Health, 42(11), 698-703. doi: 10.1111/j.1440-1754.2006.00954.x

Fletcher, O. V., May, P. A., Seedat, S., Sikkema, K. J., \& Watt, M. H. (2018). Attitudes toward alcohol use during pregnancy among women recruited from alcohol-serving venues in Cape Town, South Africa: A mixed-methods study. Social Science \& Medicine, 215, 98-106. doi:10.1016/j.socscimed.2018.09.008

France, K., Henley, N., Payne, J., D'Antoine, H., Bartu, A., O'Leary, C.,... Bower, C. (2010). Health professionals addressing alcohol use with pregnant women in Western Australia: barriers and strategies for communication. Substance Use and Misuse, 45(10), 1474-1490. doi: $10.3109 / 10826081003682172$

Haidet, P., Dains, J., Paterniti, D., Hechtel, L., Chang, T., Tseng, E., \& Rogers, J. (2002), Medical student attitudes toward the doctor-patient relationship. Medical Education, 36(6), 568-574.

Howlett, H., Gray, W. K., Dismore, L., Mackenzie, S., Langley, K., Davidson, C., \& Rankin, J. (2017). A survey of attitudes, beliefs and practice regarding alcohol use and screening in pregnancy: an opportunity for support and education? Journal of Research in Nursing, 22(8), 618-633. doi: $10.1177 / 1744987117745579$

López, M. (2013). Saber, Valorar y Actuar: Relaciones entre información, actitudes y consumo de alcohol durante la gestación. Health and Addictions/Salud y Drogas, 13(1), 35-46. doi: 10.21134/haaj.v13i1.191

Maier, S, \& West, J. (2001). Drinking patterns and alcohol-related birth defects. Alcohol Research and Health, 25(3), 168-174.

Manich, A., Velasco, M., Joya, X., García-Lara, N. R., Pichini, S., Vall, O., \& García-Algar, O. (2012). Validez del cuestionario de consumo materno de alcohol para detectar la exposición prenatal. Anales de Pediatría, 76(6), 324-328. doi:10.1016/j.anpedi.2011.09.016

Míguez, H., Magri, R., \& Suárez, M. (2009). Consumo de tabaco y bebidas alcohólicas durante el embarazo. Acta Psiquiátrica y Psicológica de América Latina, 55(2), 76-83.

Nebot, M., Cabezas, C., Marqués, F., Bimbela, J., Robledo, T., Megido, X.,...Muñoz, E. (2007). Prioridades en promoción de la salud según los profesionales de atención primaria: un estudio Delphi. Atención Primaria, 39(6), 277-331. doi: 10.1157/13106280

Organización Mundial de la Salud. (2016). Prevention of harm caused by alcohol exposure in pregnancy. Rapid review and case studies from Member States. Copenhague: WHO Regional Office for Europe.

Patton, M. (2002). Qualitative research and evaluation methods. Thousand Oaks: Sage.

Payne, J., Elliot, E., D'Antoine, H., O'Leary, C., Mahony, A., Haan, E., \& Bower, C. (2005). Health professionals' knowledge, practice and opinions about fetal alcohol syndrome and alcohol consumption in 
pregnancy. Australian and New Zealand Journal of Public Health. 29(6), 558-564.

Ritchie, J., \& Lewis, J. (2003). Qualitative research practice: A guide for social science students and researchers. Londres: SAGE Publications.

Ramos-Morcillo, A., Ruzafa-Martínez, M., Fernández-Salazar, S., del-PinoCasado R., \& Armero, D. (2014). Actitudes de médicos y enfermeras ante las actividades preventivas y de promoción en atención primaria. Atención Primaria, 46(9), 483-491. doi: 10.1016/j.aprim.2014.02.002.

Scholin, L., Hughes, K., Bellis,M.A., Eriksson, C., \& Lorna, P. (2018). "I think we should all be singing from the same hymm sheet". English and Swedish midwives' views of advising pregnant women about alcohol. Drugs: Education, Prevention and Policy, doi: $\underline{10.1080 / 09687637.2018 .1478949}$

Tough, S., Tofflemire, K., Clarke, M., \& Newburn-Cook, C. (2006). Do women change their drinking behaviors while trying to conceive? An opportunity for preconception counseling. Clinical Medicine and Research, 4(2), 97-105.

Ruiz Olabuénaga, J. I. (1996). Metodología de la investigación cualitativa. Bilbao: Ediciones de la Universidad de Deusto.

Watkins, R., Payne, J., Reibel, T., Jones, H.M., Wilkins, A., Mutch, R., \& Bower, C. (2015). Development of a scale to evaluate midwives' beliefs about assessing alcohol use during pregnancy. BMC Pregnancy Childbirth, 15(353), 1-13. doi: 10.1186/ s12884-015-0779-6 


\section{Preguntas}

\section{DATOS INICIALES:}

Profesión y área de especialización:

Sexo:

Edad:

Duración del ejercicio profesional:

PRIMERA PARTE. CONOCIMIENTO DE LAS CONSECUENCIAS DEL CONSUMO DE ALCOHOL DURANTE EL EMBARAZO, ESTÁNDARES DE ACTUACIÓN E INFORMACIÓN

- ¿Considera que hay suficiente información sobre el consumo de alcohol en el embarazo?

En caso de NO

- ¿Qué soluciones propondría?

- ¿Es malo para el feto o para la madre beber alcohol durante el embarazo?

En caso de sí

- ¿ ¿Cuáles son las posibles consecuencias?

- ¿Qué se considera un consumo de riesgo durante el embarazo? (frecuencia y cantidad)

- En las primeras entrevistas que se tiene con las embarazadas, ¿se les pregunta si beben alcohol, cuánto o con qué frecuencia? ¿Está estandarizado?

En caso de que sí se pregunte

- Si la mujer admite beber alcohol

- ¿ ¿Qué medidas se toman? ¿Qué se les dice?

- ¿ ¿Se piden analíticas concretas que permitan ver si el alcohol está generando algún problema?

- ¿ ¿Se le ofrece alguna ayuda o alternativa? ¿Hay algún procedimiento estandarizado para estos casos?

- Si la mujer dice que no bebe alcohol

- ¿ ¿Se le pregunta más veces en sucesivas consultas?

- ¿ ¿Se incide en momentos puntuales o en ocasiones especiales?

- ¿Se informa a las embarazadas de que las bebidas "sin alcohol" (como la cerveza "sin") tienen un pequeño porcentaje de alcohol?

- $\quad$ ¿Se le pregunta por sus hábitos de bebida previos al embarazo? (frecuencia y cantidad)

- ¿ ¿ ¿Se le pregunta si le está suponiendo algún problema o dificultad no beber?

En caso de NO se pregunte

- Motivos para no preguntar más.

SEGUNDA PARTE: OPINIONES Y CREENCIAS DE LA GESTANTE RESPECTO AL CONSUMO DE ALCOHOL DURANTE EL EMBARAZO PERCIBIDAS POR LOS PROFESIONALES

- ¿Qué piensan las embarazadas sobre el consumo de alcohol durante el embarazo? ¿Tienen creencias erróneas o distorsionadas?

- ¿Conocen el TEAF?

TERCERA PARTE: DIFICULTADES AL ABORDAR EL TEMA EN CONSULTA

- Problemas a la hora de hablar del consumo de alcohol con las embarazadas.

- Al preguntar a las embarazadas por su consumo de alcohol:

- ¿Cuál es la reacción de ellas?

- ¿Cómo reaccionan las que admiten beber? (Sentimientos de culpa, vergüenza, no lo consideran un problema, consideran necesitar ayuda, etc.)

SUGERENCIAS

- ¿Tiene alguna sugerencia sobre cambios que se podrían hacer para facilitar el acceso de las embarazadas a la información sobre el consumo de alcohol durante el embarazo?

- ¿Y para mejorar la comunicación respecto a este tema? 
ANEXO 2. Codificación del discurso: categorías NVivo

\begin{tabular}{|c|c|c|}
\hline $1^{\text {a }}$ Generación & 2a Generación & $3^{a}$ Generación \\
\hline \multirow{12}{*}{$\begin{array}{l}\text { 1. Conocimiento de las } \\
\text { consecuencias del consumo de } \\
\text { alcohol durante el embarazo }\end{array}$} & \multirow[t]{4}{*}{ 1.1 Consumo de riesgo } & 1.1.1 Considera que todo consumo en el embarazo es de riesgo \\
\hline & & 1.1.2 Desconoce el consumo de riesgo \\
\hline & & 1.1.3 Diferencia entre tipos de alcohol más/menos nocivos \\
\hline & & $\begin{array}{l}\text { 1.1.4 Diferencia entre cantidades y frecuencia de consumo para establecer el } \\
\text { riesgo }\end{array}$ \\
\hline & \multirow[t]{3}{*}{ 1.2 Recomendaciones oficiales } & 1.2.1 Las conoce, pero no las aplica \\
\hline & & 1.2.2 Las conoce y las aplica \\
\hline & & 1.2.3 Ni las conoce ni las aplica \\
\hline & \multirow[t]{3}{*}{1.3 Consecuencias negativas } & 1.3.1 En el feto \\
\hline & & 1.3.2 En la madre \\
\hline & & 1.3.3 En ambos \\
\hline & \multirow{2}{*}{$\begin{array}{l}\text { 1.4 Considera que hay suficiente } \\
\text { información sobre el tema }\end{array}$} & 1.4.1 Sí \\
\hline & & 1.4.2 No \\
\hline \multirow{12}{*}{$\begin{array}{l}\text { 2. Estándares de actuación e } \\
\text { información a las gestantes }\end{array}$} & \multirow{4}{*}{$\begin{array}{l}2.1 \text { Se pregunta sobre el consumo de } \\
\text { alcohol }\end{array}$} & 1.4.3 Soluciones para incrementar la información \\
\hline & & 2.1.1 Se sigue un protocolo estandarizado \\
\hline & & 2.1.2 Se pregunta, incluyendo la frecuencia y/o cantidad \\
\hline & & 2.1.3 Sólo en la primera consulta \\
\hline & \multirow[t]{4}{*}{ 2.2 No se pregunta } & 2.2.1 No hay un protocolo estandarizado o lo desconoce \\
\hline & & 2.2.2 Se pregunta por el consumo de tóxicos en general \\
\hline & & 2.2.3 Se centran en el consumo de tabaco \\
\hline & & 2.2.4 Son más permisivos que con otras sustancias tóxicas \\
\hline & \multirow[t]{4}{*}{ 2.3 Si la gestante admite beber } & 2.2.5 No pregunta \\
\hline & & 2.3.1 Aconseja que no beba \\
\hline & & 2.3.2 Explora los motivos de consumo \\
\hline & & 2.3.3 Ofrece ayudas o alternativas \\
\hline \multirow{5}{*}{$\begin{array}{l}\text { 3. Conocimiento que tienen y } \\
\text { actitud de las gestantes cuando } \\
\text { se plantea el tema }\end{array}$} & \multirow[t]{3}{*}{ 3.1. Tipo de reacción } & 2.3.4 Indica la inexistencia de procedimientos estandarizados \\
\hline & & 3.1.1 Con naturalidad \\
\hline & & 3.1.2 "A la defensiva" \\
\hline & 3.2 Conocen efectos y consecuencias & 3.1.3 Mienten \\
\hline & \multirow[t]{4}{*}{ 4.1 Tienen dificultades } & 3.2.1 Sí \\
\hline 4. Dificultades de los & & 3.2.2 No \\
\hline profesionales ante el abordaje & & 4.1.1 Temen emitir juicios de valor \\
\hline \multirow[t]{5}{*}{ del consumo de alcohol } & & 4.1.2 Temen encontrase con casos de alcoholismo \\
\hline & 4.2 No presentan dificultades & 4.1.3 Se quejan de la falta de tiempo en consulta \\
\hline & 5.1 Educación para la salud & 4.1.4 Consideran que es un tema tabú \\
\hline & \multirow{2}{*}{$\begin{array}{l}5.2 \text { Información específica a las } \\
\text { gestantes }\end{array}$} & 4.2.1 Lo abordan con naturalidad \\
\hline & & 5.1.1 Campañas en los medios de comunicación \\
\hline \multirow[t]{6}{*}{ 5. Sugerencias de mejora } & \multirow{4}{*}{$\begin{array}{l}5.3 \text { Mejoras en la comunicación } \\
\text { profesional sanitario/gestante }\end{array}$} & 5.2.1 Trípticos \\
\hline & & 5.2.2 Avisos en las bebidas \\
\hline & & 5.2.3 Desde el ámbito sanitario \\
\hline & & 5.3.1 Generar relación de confianza con la embarazada \\
\hline & \multirow{2}{*}{$\begin{array}{l}\text { 5.4 Aumentar la concienciación de los } \\
\text { profesionales sanitarios }\end{array}$} & 5.3.2 No juzgar \\
\hline & & 5.3.3 Formar a los profesionales en técnicas de comunicación \\
\hline
\end{tabular}

